\title{
Le métier d'enseignant en période de réformes
}

Le cas anglais

Teaching at the crossroads, education reform in the UK

La docencia en período de reformas. El caso inglés

\section{Marilyn Osborn}

\section{OpenEdition}

\section{Journals}

Édition électronique

URL : https://journals.openedition.org/ries/1852

DOI : 10.4000/ries. 1852

ISSN : 2261-4265

\section{Éditeur}

France Education international

\section{Édition imprimée}

Date de publication : 1 septembre 2002

Pagination : $27-40$

ISBN : 978-285420-554-1

ISSN : $1254-4590$

\section{Référence électronique}

Marilyn Osborn, "Le métier d'enseignant en période de réformes », Revue internationale d'éducation de Sèvres [En ligne], 30 | septembre 2002, mis en ligne le 24 novembre 2011, consulté le 06 juillet 2021. URL : http://journals.openedition.org/ries/1852 ; DOI : https://doi.org/10.4000/ries.1852 


\section{Le métier d'enseignant en période de réformes}

\section{Le cas anglais}

\section{Marilyn Osborn}

Faire la liste ${ }^{1}$ des obligations de service des enseignants en Angleterre ne suffit pas à représenter l'ampleur des réformes qui ont bouleversé la vie des enseignants en Angleterre au cours de ces quatorze dernières années. Cet article tente d'analyser les principaux changements intervenus dans la relation des enseignants du primaire à leur travail et à leurs pratiques pédagogiques après la récente politique de réformes et de comprendre leurs répercussions sur les enseignants, leurs élèves, leurs établissements ainsi que sur le système éducatif et sur la société dans son ensemble.

Les données citées dans cet article sont toutes extraites de $\mathrm{PACE}^{2}$, un projet de recherche mené au niveau national auprès de cent cinquante enseignants et directeurs d'établissements, dans quarante-huit établissements de huit communes anglaises, pendant les huit années qui ont suivi la mise en place des grandes réformes éducatives. L'enquête a été menée auprès de tous les enseignants et cinquante-quatre d'entre eux ont été suivis plus attentivement dans leur classe tout au long de l'enquête. Trois grandes questions étaient formulées au départ :

- quels changements les enseignants du primaire ont-ils pu observer dans leur compréhension et leur définition de leur métier et de leurs responsabilités professionnelles?

- en quoi leurs pratiques professionnelles en ce qui concerne le curriculum, la pédagogie et l'évaluation ont-elles changé?

- en quoi la politique nationale a-t-elle été influencée par les attentes, les cultures et les comportements des enseignants, et en particulier leur perception des responsabilités professionnelles?

\section{LE CONTEXTE des RÉFORMES}

Les spécialistes de l'éducation des autres pays sont souvent étonnés de l'absence de tradition de curriculum national en Angleterre et au Pays de Galles jusqu'à l'adoption de l'Education Reform $\mathrm{Act}^{3}$ en 1988. Historiquement, l'Angleterre

\footnotetext{
1. Voir pp. 30-31.

2. PACE : Primary Assessment, Curriculum and Experience Project : évaluation, programme et projet expérimental pour le primaire.

3. Loi de réforme éducative.
} 
a été presque le seul pays à ne pas avoir de curriculum national. Les enseignants, en particulier ceux de l'enseignement primaire et secondaire, ont pu jouir d'une liberté considérable en termes de contenus comme de pratiques pédagogiques puisqu'ils n'étaient encadrés par aucune instruction relative aux programmes ou aux examens. Tout a changé radicalement en 1988, avec la mise en place de l'Educational Reform Act, une initiative politique d'une ambition et d'une portée sans précédent qui visait à relever le niveau d'exigences des enseignants quant aux résultats des élèves. L'introduction du National Curriculum ${ }^{4}$ a été accompagnée de la mise en place d'un système d'évaluation unique et commun à tous les élèves qui prévoit des évaluations nationales à l'âge de sept, onze, quatorze et seize ans. Ces réformes et les nombreux changements qu'elles ont entraînés ont profondément modifié la façon dont les instructions officielles définissaient le rôle et les pratiques des enseignants du primaire. Ces réformes comprenaient l'introduction de nouveaux modes de gestion des établissements, de nouveaux modes d'évaluation du travail des enseignants et l'apparition de la notion de marché dans le secteur éducatif.

Après son arrivée au pouvoir en mai 1997, le gouvernement travailliste a publié le White Paper Excellence in Schools ${ }^{5}$, manifestant ainsi son intention de poursuivre la nouvelle politique éducative. Cependant, le projet a été réorienté et les priorités ont changé. On a accordé une place importante à l'intégration sociale et insisté sur l'apprentissage de base de l'arithmétique et de la maîtrise de la langue. En 1999, un projet de révision du National Curriculum a été publié. Ce curriculum 2000 témoignait d'une meilleure concertation avec les enseignants que les versions précédentes. Cependant, le curriculum n'a pas été aussi «allégé» que prévu, et l'espace laissé aux enseignants pour mettre en pratique leur point de vue professionnel est toujours aussi réduit.

Le parti travailliste a également renforcé le cadrage pédagogique en imposant une structure horaire obligatoire dans les écoles primaires pour l'apprentissage de l'arithmétique et de la maîtrise de la langue. Il a par ailleurs confié à l'OFSTED ${ }^{6}$ la mise en place d'un système d'inspection des écoles primaires plus exigeant, qui a entraîné la «stigmatisation» des établissements en difficulté. Ces mesures ont été ensuite renforcées par le développement progressif de la notion d'objectifs à atteindre pour les établissements et pour les administrations locales ainsi que par l'introduction d'une corrélation, par le biais de la notion de "gestion par résultats", entre les résultats d'une classe et l'amélioration du salaire de l'enseignant. Dans l'ensemble, ces différentes initiatives ont eu pour résultat de soumettre l'enseignement dans le primaire à des exigences et à des pressions extérieures à l'école de plus en plus fortes. Paradoxalement, on a

4. National Curriculum : curriculum national.

5. Livre Blanc sur la qualité de l'école.

6. OFSTED : Office for Standards in Education. 
donné aux écoles primaires de plus en plus d'autonomie dans la gestion de leurs propres budgets. Contrairement à la plupart des autres pays européens, les écoles sont libres de recruter leurs enseignants et le personnel administratif bien que ces derniers soient salariés de la LEA ${ }^{7}$. Quel est l'impact de ces nombreuses initiatives sur le travail et l'identité professionnelle des enseignants?

\section{UN DÉFI EN TERMES DE PROFESSIONNALISME, DE TRAVAIL ET DE VALEURS}

L'enquête PACE (Osborn, Broadfoot, McNess et al., 2000) montre que les enseignants se sont sentis submergés par «l'avalanche» de mesures gouvernementales prises à la fin des années 1980 et au cours des années 1990. Leurs réponses laissent entendre qu'ils se sont sentis littéralement assaillis de critiques et d'exigences extérieures - en particulier celles des parents - quant à leurs responsabilités, alors qu'ils gardaient un sens profond de leur engagement moral vis-à-vis de leurs élèves. Beaucoup d'enseignants se sont sentis très angoissés par une prolifération incessante d'exigences extérieures alors que leur espace personnel et leur capacité d'initiatives se voyaient considérablement réduits. Pour nombre d'entre eux, l'accroissement de la pression et des contraintes a été d'autant plus difficile à supporter qu'ils étaient convaincus que ce qu'on leur demandait n'était pas souhaitable en termes éducatifs et, même, n'allait pas dans le sens de l'intérêt des élèves. L'élévation continuelle du niveau attendu et le contrôle externe des évaluations nationales ont été l'une des principales causes de ce conflit. Pour certains enseignants, en particulier ceux qui travaillaient avec des élèves de milieux socialement et économiquement défavorisés, le curriculum national était en soi une cause d'angoisse et de frustration car il était incapable de répondre aux besoins spécifiques de leurs élèves.

Des changements significatifs ont également touché le travail et les valeurs des enseignants (Acker, 1999; Campbell, 1996; Troman, 1996; Webb et Vulliamy, 1996; Woods et al., 1997). L'enquête PACE a montré que beaucoup d'enseignants se sont résignés à se sentir de plus en plus instrumentalisés lorsque contenus et méthodes leur ont été imposés. Dans les médias, les enseignants faisaient l'objet d'une vague de critiques et de ce que Ball (1994) a appelé un «discours de la dérision». Nombre d'entre eux ont perçu ce basculement de leur autonomie professionnelle vers un engagement fondé sur une responsabilité contractuelle comme une source d'anxiété croissante, d'un conflit de valeurs et d'une diminution de leur satisfaction professionnelle. Par-dessus tout, ils se sont sentis liés par une exigence de résultats. Par ailleurs, ils ont eu l'impression que leur attachement "affectif» à l'enseignement - leur sens de la vocation et

7. Local Education Authority: autorité locale de l'éducation. 


\section{Les conditions d'accès au métier d'enseignant}

Les textes officiels qui définissent le statut d'enseignant qualifié et les critères requis pour suivre la formation initiale au métier d'enseignant stipulent que tous les candidats à cette formation doivent avoir obtenu au minimum la note $\mathrm{C}$ à leur GCSE ${ }^{8}$ d'anglais et de mathématiques. On exige également de certains candidats la même note à un GCSE dans une matière scientifique. En outre, tous les candidats doivent être titulaires d'une licence (ou équivalent) avant ou pendant leur formation. Les trois principaux cursus qui conduisent à l'enseignement sont un premier cycle universitaire ${ }^{9}$ enrichi d'un diplôme ${ }^{10}$ de deuxième cycle en sciences de l'éducation, le bachelor en sciences de l'éducation, enfin, les cursus fondés sur le parcours professionnel, le registered teacher programme ${ }^{11}$ et le graduate teacher programme ${ }^{12}$. Ce sont des programmes pour les enseignants non qualifiés qui peuvent ainsi obtenir un diplôme ${ }^{10}$ de deuxième cycle en sciences de l'éducation tout en travaillant comme enseignant. Dans ce cas, les candidats doivent être âgés de 24 ans au moins, pouvoir justifier d'un emploi dans un établissement, et présenter une recommandation institutionnelle. Tous les candidats doivent également voir leur stage d'un an dans un établissement validé et sont testés sur des compétences de base - arithmétique, maîtrise de la langue, technologies de communications et d'information - avant d'obtenir le statut d'enseignant qualifié.

\section{Les responsabilités professionnelles des enseignants}

Le School Teachers' Pay and Conditions of Employ Document $2000^{13}$ énumère les obligations de service des enseignants.

\section{- Enseignement (en fonction du curriculum de l'établissement)}

Organiser et préparer les cours et les leçons; enseigner en fonction d'un public d'élèves donné (selon les besoins des élèves), organiser et noter dans le cahier de textes le travail à faire en classe et à l'extérieur; évaluer régulièrement le développement, les progrès et les acquisitions des élèves et en rendre compte.

\section{- Autres activités pédagogiques}

Favoriser les progrès et l'épanouissement de chaque élève, de chaque classe ou de chaque groupe d'élèves dont l'enseignant a la responsabilité; guider et conseiller les élèves sur les questions éducatives ou sociales, ainsi qu'en ce qui concerne leur orientation et leur future carrière ( $\mathrm{y}$ compris en indiquant où trouver des informations plus spécialisées dans certains domaines); noter ses observations et faire un rapport; noter les besoins personnels et sociaux de ses élèves et faire un rapport; dialoguer avec les parents d'élèves et recueillir leur avis; dialoguer et coopérer avec les personnes ou les institutions extérieures à l'établissement; participer aux réunions organisées sur chacun des sujets précédents.

8. GCSE : General Certificate of Secundary Education: examen passé à l'âge de 16 ans sanctionnant les connaissances de l'élève dans une matière.

9. L'enseignement supérieur britannique se découpe en un premier cycle appelé undergraduate course qui aboutit à l'obtention, en trois ou quatre années, du bachelor's degree, comparable à la licence en France. Ce diplôme conditionne l'accès au deuxième cycle qui s'intitule graduate ou postgraduate studies.

10. Certificate en anglais, terme correspondant à un diplôme postgraduate (puisqu'il est nécessaire pour s'inscrire de posséder un degree, éventuellement dans une autre discipline). Source: the British Council http://www.britishcouncil.org/france/french/education/description.htm

11. Il est accessible après deux années d'études supérieures validées. Source: the British Council http://www.britishcouncil.org/france/french/education/enseigner.htm\#registered

12. Il est accessible après trois années d'études supérieures. Source : the British Council http://www. britishcouncil.org/france/french/education/enseigner.htm\#registered

13. School Teacher's Pay and Conditions of Employment Document 2000: circulaire sur la rémunération et les conditions d'emploi des enseignants 2000. 


\section{- Évaluation}

Participer à l'élaboration des critères d'évaluation.

\section{- Actualisation des connaissances, formation continue}

Renouveler ses méthodes d'enseignement et son programme de travail (de temps en temps); participer au dispositif de formation continue de développement professionnel; participer à l'accueil et l'adaptation des nouveaux collègues.

\section{- Méthodes d'enseignement}

Donner son avis et travailler en coopération avec le responsable des études et les autres enseignants à la préparation et à l'organisation des cours (outils pédagogiques, programmes, méthodes d'enseignement et d'évaluation, enseignement religieux).

\section{- Discipline, santé et sécurité}

Maintenir l'ordre et la discipline en classe; veiller sur la santé et sur la sécurité des élèves lorsqu'ils sont dans l'enceinte de l'établissement ainsi qu'à l'extérieur dans le cadre d'une activité scolaire autorisée.

\section{- Réunions du personnel}

Se rendre aux réunions organisées sur le curriculum de l'établissement, l'administration ou l'organisation de l'établissement, y compris en matière de religion.

\section{- Remplacement d'un enseignant absent}

Surveiller les élèves d'un enseignant absent et faire cours à chaque élève «dans la mesure du possible».

\section{- Examens nationaux}

Participer à la préparation des élèves aux examens nationaux et les évaluer dans cette perspective; faire un compte rendu écrit de cette évaluation; participer aux dispositions prises pour présenter les élèves aux examens et surveiller les épreuves.

\section{- Gestion}

Participer au recrutement et à l'évolution professionnelle d'autres enseignants et membres du personnel, y compris pour l'accueil et l'évaluation des nouveaux enseignants; coordonner ou organiser le travail d'autres enseignants; participer si nécessaire au renouvellement, au développement et à l'organisation des activités en relation avec le curriculum, l'organisation et à l'enseignement religieux; aider le responsable de l'établissement à repenser les évaluations (dans le cas des enseignants qui ont des responsabilités administratives).

\section{- Administration}

Participer à l'organisation et à l'administration qui découlent des responsabilités décrites précédemment, y compris l'encadrement de personnels susceptibles d'assister les enseignants dans l'établissement ainsi que la répartition et l'affectation de matériels et d'équipements; assister aux réunions, faire l'appel et encadrer les élèves avant, pendant ou après chaque cours; pour un enseignant à temps plein, être disponible 195 jours par an, dont 190 sont susceptibles d'être consacrés à l'enseignement; effectuer 1265 heures de "temps dirigé» par an (selon les directives du responsable de l'établissement); travailler éventuellement «autant qu'il le faut pour remplir ses obligations professionnelles le plus efficacement possible». Ces heures supplémentaires comprennent la correction des copies, la rédaction de comptes rendus sur les élèves, la préparation des leçons, des outils pédagogiques et du programme des cours. Au sein de ces 1265 heures de temps dirigé, la répartition entre temps d'enseignement et temps réservé aux autres tâches relève du responsable de l'établissement ${ }^{14}$.

14. Adapté de Document Summary Service, University of Bristol 2000. 
de l'investissement personnel - était mis en cause par une volonté de les voir devenir des "techniciens spécialisés» dans la transmission de savoirs et de compétences prédéfinis (Hargreaves, 1999; Jeffrey, 1999; Nias, 1989). Considérer, comme l'avaient fait plusieurs gouvernements successifs, l'éducation comme un "produit» susceptible d'être une fourniture quantifiable était à l'opposé d'une conception, partagée par de nombreux enseignants, de l'éducation se donnant pour objectif l'épanouissement personnel.

\section{LES RÉPONSES STRATÉGIQUES DES ENSEIGNANTS}

La plupart des enseignants considéraient que les effets les plus néfastes des réformes décrites ci-dessus étaient véhiculés par le développement excessif des notions de collaboration et de collégialité. Cette façon de travailler avait pourtant été souvent l'une des forces de l'enseignement primaire (Nias et al., 1989). En effet, au début des années 1990, les enseignants ressentaient de plus en plus le besoin de travailler ensemble pour faire face à ces nouveaux défis et à leurs répercussions. Ainsi, la préparation du curriculum, la coordination de l'établissement dans son ensemble, la préparation à l'inspection et la communication externe, de même que l'enseignement, étaient définis par des enseignants qui mettaient leurs connaissances et leurs compétences en commun. Parfois les dispositions prises étaient extrêmement créatives et enrichissantes. L'équipe, en totalité ou en partie, éprouvait un sentiment nouveau de fierté professionnelle.

D’autres études, comme celle de Richards (1998), montrent comment les petites écoles primaires de campagne ont procédé à une «appropriation tranquille» du National Curriculum et ont adapté avec pragmatisme les instructions officielles à leur cas particulier. Elles ont mieux compris l'évaluation et l'expertise (Craft, 1996; Gipps et al., 1995; Torrance et Pryor, 1998). L'étude de Nixon et al. (1997) montre également l'émergence d'un «nouveau professionnel» dont les valeurs et les pratiques reflètent une intégration créative des nouvelles exigences avec des valeurs professionnelles fondamentales. Hargreaves (1994) l'explique par le développement de l'organisation. D'autres études, comme celle de Woods (1995) et celle de Woods et Jeffrey (1996), ont mis en évidence le talent et la créativité des enseignants lorsqu'il s'agit de protéger leurs valeurs, leur imagination et leur engagement vis-à-vis des élèves en dépit du National Curriculum et des autres exigences.

Cependant, dans certains cas, une gestion plus autoritaire de l'établissement engendre une "collégialité contrainte» (Hargreaves, 1991) et un engagement des enseignants plus contractuel que professionnel, en particulier lorsque la collaboration était imposée d'en haut et accordait plus d'importance à la paperasserie et à la rédaction de rapports qu'à un travail commun fondé sur l'enseignement. D'autres, comme Menter et al. (1996), ont mis en relation l'érosion de l'engagement des enseignants du primaire avec leur nouveau posi- 
tionnement et leur instrumentalisation dans le cadre d'un processus où la gestion du travail devient plus importante.

Partout, il semble que, lorsque l'enseignant, ou l'établissement dans son ensemble, manquait de confiance en soi pour aménager de façon «créative» les instructions officielles, ou lorsque les circonstances privées ou personnelles ne le permettaient pas, il y eut conflit, anxiété et désillusions. Ainsi, tandis que certains enseignants se montraient capables de réagir avec créativité à la stimulation d'un nouvel environnement pédagogique, d'autres, pour des raisons souvent liées à leurs «histoires de vie » personnelles ou aux problèmes posés par l'admission de certains élèves, furent accablés et démoralisés. Ce fut souvent le cas d'enseignants plus âgés et plus expérimentés qui s'opposaient par principe aux nouvelles dispositions ou qui trouvaient difficile de transformer leurs valeurs professionnelles et leurs pratiques pédagogiques. Quoi qu'il en soit, ce qui a fait la différence dans la capacité de s'adapter au changement, cela a été la confiance que chacun pouvait avoir, non seulement en ses compétences et en ses savoirs, mais également en soi-même. Certains n'ont tout simplement pas pu s'adapter à la demande qui leur était faite de couvrir un champ plus vaste de connaissances. D’autres, compétents, cultivés, dévoués et pleins de bonne volonté, en ont eu assez de lutter ou n'ont pas souhaité faire de compromis. Beaucoup d'enseignants et de responsables d'établissement expérimentés ont pris une retraite anticipée ou ont abandonné la profession pour raisons médicales. Comme l'indiquent les conclusions de notre enquête, tandis que la profession se remodelait grâce à de nouvelles modalités de formation, d'évaluation et de rémunération, les enseignants les plus jeunes ou plus récemment formés se sont adaptés aux structures et aux exigences nouvelles et ont commencé à "prendre les choses comme elles étaient».

\section{LeS PRATIQUes de CLASSE}

Le poids de cette relation étroite entre directives officielles du Curriculum national et discours public général d'une part, évaluation nationale et exigences de l'OFSTED en matière d'inspection de l'autre (Jeffrey and Woods, 1996), laissait aux enseignants et aux établissements peu d'espace pour redéfinir ce qu'ils devaient enseigner, quand et selon quel modèle. En outre, on ne cessait de refuser aux enseignants et aux élèves la plus petite initiative personnelle en matière d'horaire, de volume et de contrôle des contenus d'enseignement. De fait, la réduction progressive de l'autonomie des enseignants et des élèves est sans doute le résultat le plus significatif de la politique initiée par l'Education Reform Act de 1988.

L'enquête montre, chez les enseignants, un net basculement de leur adhésion antérieure à des modèles d'apprentissage «constructivistes » vers une nouvelle attitude induite par une perception de l'enseignement/apprentissage comme une livraison/assimilation d'un ensemble de connaissances prédéfinies. 
Quoiqu'il ne faille pas méconnaître les éléments de continuité avec le passé (Alexander, 1997), notre étude montre que les pratiques de classe ont profondément changé.

On peut présenter les résultats de l'enquête au niveau de la salle de classe en fonction des trois principaux «systèmes de communication» qui existent dans les établissements : le curriculum, la pédagogie et l'évaluation. Tout d'abord, le curriculum a été de plus en plus «cloisonné», dans le sens d'une fragmentation explicite entre les différentes matières (Bernstein 1996). Ensuite, les classes ont été de plus en plus fortement "encadrées", c'est-à-dire que les enseignants ont vu leur liberté de choix dans leur façon d'enseigner diminuer progressivement et ce mode de fonctionnement s'est répercuté sur les élèves. Enfin, l'évaluation s'est imposée de façon de plus en plus normative, avec des exigences toujours plus élevées au fur et à mesure que les attentes en matière de responsabilités et de résultats étaient formulées avec plus de force et de précision.

Les conséquences potentielles de cette tendance vers un enseignement global pour toute la classe, vers l'exécution des directives par les enseignants, vers la définition d'un emploi du temps précis par sujet abordé et vers la capacité à fonctionner en groupe furent en outre renforcées par un système d'évaluation qui instrumentalisait de plus en plus les résultats et faisait pencher la balance éducative en faveur de la connaissance plutôt que de la dimension affective, accordant plus d'importance au produit qu'au processus. Résultat: dans la classe, la pression est de plus en plus forte, tout étant focalisé sur les résultats de l'élève (Osborn et al., 2000).

Il serait faux de croire, cependant, que la réforme n'a concerné que l'adaptation des enseignants à des exigences venues d'en haut. Les directives gouvernementales n'ont pas été transférées du jour au lendemain au sein de l'école et de la classe. Elles sont plutôt le fruit d'une série d'interprétations et de réinterprétations successives par les différents acteurs du système (Ball, 1994). L'enquête d'Osborn et al. (2000) met aussi en évidence la façon dont les enseignants sont devenus à leur tour des «réformateurs de terrain» et se sont efforcés, notamment, de protéger leurs élèves de ce qui, pour eux, était le plus néfaste dans les réformes récentes. L'enquête analyse une série de réformes et de stratégies menées au niveau des établissements.

Lorsqu'on tente de comprendre la signification des différentes réponses apportées par les enseignants aux récentes initiatives gouvernementales, on s'aperçoit que la question de la motivation professionnelle est essentielle. L'enquête PACE montre le passage progressif d'un professionnalisme fondé sur la notion d'engagement, lié à une satisfaction intrinsèque, à une motivation contractuelle fondée sur les résultats, guidée par une exigence externe de responsabilité et d'évaluation. Nous formulons l'hypothèse que cela pourrait entraîner, chez les enseignants, un déclin de leur sens personnel des responsabilités et de leur engagement moral. Bien qu'en grande partie difficiles à cerner, ces aspects 
du professionnalisme sont néanmoins très importants et ont une répercussion significative sur la qualité de l'expérience vécue en classe (Pollard et al., 2000).

Le tableau qui suit résume le débat sur la signification des directives gouvernementales mises en place en Angleterre à la fin des années 1980 et au cours des années 1990, en ce qui concerne la nature et la qualité de la motivation professionnelle et des pratiques des enseignants. Les concepts sont empruntés à Basil Bernstein qui, bien qu'il se soit principalement intéressé aux élèves, oppose une "compétence» fondée sur un modèle pédagogique où l'enseignant est un médiateur et où l'élève se comporte en acteur créatif, à une

Tableau ${ }^{\circ} 1$

Quelques aspects contradictoires des modèles de compétence et de performance selon les écoles et les enseignants

\begin{tabular}{|c|c|c|}
\hline $\begin{array}{c}\text { Type } \\
\text { de modèle }\end{array}$ & $\begin{array}{l}\text { «MODÈLE DE COMPÉTENCE» } \\
\text { Éducation progressive libérale } \\
\text { "Gestion invisible» avec une } \\
\text { certaine autonomie professionnelle }\end{array}$ & $\begin{array}{l}\text { «MODĖLE DE PERFORMANCE " } \\
\text { Éducation de performance } \\
\text { "Gestion visible» avec un } \\
\text { certain contrôle professionnel }\end{array}$ \\
\hline $\begin{array}{l}\text { Mode } \\
\text { d'organisation }\end{array}$ & $\begin{array}{l}\text { Professionnel, peu encadré. } \\
\text { Moyens de contrôle : auto régulation, } \\
\text { socialisation et règles internes. }\end{array}$ & $\begin{array}{l}\text { Mécanique, doté d'une structure } \\
\text { hiérarchique et bureaucratique. } \\
\text { Réglementation du contrôle } \\
\text { et de la coordination. }\end{array}$ \\
\hline $\begin{array}{l}\text { Style } \\
\text { de gestion }\end{array}$ & $\begin{array}{l}\text { Collégial : on insiste sur les capacités, } \\
\text { le dialogue et le consensus. } \\
\text { Relations informelles. }\end{array}$ & $\begin{array}{l}\text { Directif: on insiste sur l'efficacité } \\
\text { et on fixe des objectifs de résultats. } \\
\text { Relations plus formelles. }\end{array}$ \\
\hline $\begin{array}{l}\text { Rôle } \\
\text { des enseignants }\end{array}$ & $\begin{array}{l}\text { Les enseignants sont des médiateurs, } \\
\text { la dimension affective est intrinsèque } \\
\text { au rôle d'enseignant. }\end{array}$ & $\begin{array}{l}\text { Les enseignants sont des éducateurs } \\
\text { et des évaluateurs, l'accent est mis } \\
\text { sur les compétences cognitives } \\
\text { et sur l'encadrement. }\end{array}$ \\
\hline $\begin{array}{l}\text { Professionnalisme } \\
\text { des enseignants }\end{array}$ & $\begin{array}{l}\text { Engagement professionnel fondé } \\
\text { sur la confiance et l'attachement } \\
\text { à l'enseignement comme forme } \\
\text { de développement personnel. } \\
\text { Confiance en soi, épanouissement } \\
\text { et spontanéité dans l'enseignement. }\end{array}$ & $\begin{array}{l}\text { Professionnalisme pour remplir } \\
\text { un contrat où l'on s'engage à délivrer } \\
\text { un enseignement perçu comme } \\
\text { un produit par les individus, et comme } \\
\text { une nécessité nationale pour la croissance } \\
\text { économique. Moins de confiance, } \\
\text { d'épanouissement et de spontanéité } \\
\text { dans la manière d'enseigner. }\end{array}$ \\
\hline $\begin{array}{l}\text { Responsabilité } \\
\text { des enseignants }\end{array}$ & Responsabilité «morale» et personnelle. & $\begin{array}{l}\text { Responsabilité contractuelle et externe } \\
\text { renforcée par l'inspection. }\end{array}$ \\
\hline $\begin{array}{l}\text { Coordination } \\
\text { générale } \\
\text { de l'établissement }\end{array}$ & $\begin{array}{l}\text { Autonomie relative et collaboration } \\
\text { informelle des enseignants. }\end{array}$ & $\begin{array}{l}\text { Organisation scolaire formelle } \\
\text { et collégialité « dirigée». }\end{array}$ \\
\hline Coûts & $\begin{array}{l}\text { Onéreux car la formation des enseignants } \\
\text { est complexe et les pratiques scolaires } \\
\text { consommatrices de temps. }\end{array}$ & $\begin{array}{l}\text { Moins onéreux car la formation } \\
\text { des enseignants est plus explicite et } \\
\text { les pratiques scolaires sont systématisées. }\end{array}$ \\
\hline
\end{tabular}


"performance» qui privilégie une production spécifique de l'élève, l'acquisition de compétences spécialisées, et la rédaction par l'élève du texte précis qu'on lui demande d'élaborer. Les modalités de l'évaluation leur ayant été présentées de façon explicite, les élèves savent ce qui sera évalué. En retour, leurs résultats permettront de les classer de façon hiérarchique.

Cependant, il apparaît clairement, d'après ce que je viens d'exposer, que les enseignants sont à leur tour soumis à un modèle de "performance » du même ordre. Comme pour les élèves, le choix de ce modèle est une stratégie globale qui inclut les apports, les processus d'acquisition et les productions du système éducatif. Au cours de ces dernières années, on a vu se renforcer au niveau national la spécification de l'ensemble des compétences proposées dans la formation initiale des professeurs et par conséquent de ce qui venait "approvisionner» le système éducatif. Là encore, les pouvoirs de l'OFSTED se sont étendus avec obligation de passer par un système d'inspection global qui s'appuie sur une définition de ce qui constitue la "qualité» dans les "processus» d'enseignement. Enfin et surtout, les enseignants sont soumis à une définition externe de leur «rendement» par la publication de tableaux de résultats des élèves. En outre, comme je l'ai déjà évoqué, les conditions de rémunération des enseignants et l'organisation de leur carrière ont fait l'objet de réformes radicales à partir de l'idée qu'il est possible et souhaitable de juger les résultats d'un enseignant en fonction de critères explicites. On exige des enseignants, comme des élèves, d'évoluer dans un système d'enseignement de plus en plus orienté vers la «performance» et fondé sur des critères de qualité externes. La vie professionnelle des enseignants et l'expérience scolaire des élèves sont, de plus en plus, l'objet d'évaluations "péremptoires» et normatives. Le tableau 1 oppose certains de ces aspects pour faciliter l'analyse, bien que la situation soit en réalité bien plus complexe.

\section{VERS UN NOUVEAU PROFESSIONNALISME DES ENSEIGNANTS DE PRIMAIRE?}

L'enquête PACE analyse les effets d'une conception de plus en plus gestionnaire du métier d'enseignant en Angleterre. Elle met en évidence l'impact des pressions sur l'évolution du rôle de l'enseignant, qui passe du statut de semi-professionnel relativement autonome à celui de technicien spécialisé. Les récentes réformes en Angleterre ont contraint les enseignants à s'adapter à un système d'éducation orienté vers la "performance» et fondé sur des critères de qualité externes. La vie professionnelle des enseignants est de plus en plus soumise à des évaluations "péremptoires» et formelles. Il faut reconnaître que ces tensions ne sont pas propres à ce seul petit pays, même si elles y sont particulièrement sensibles. Comme l'a suggéré Sultana (1994) dans son analyse du travail des enseignants dans une Europe unifiée, «le souci de la 
"performance" dans le domaine de l'éducation a entraîné une prédominance de ce qu'Habermas appelle la "rationalité instrumentale" ", selon laquelle les critères de choix de la meilleure méthode d'action sont déterminés, non pas en fonction des raisons les plus pertinentes, mais en fonction du moyen le plus efficace d'atteindre les objectifs souhaités. La rationalité instrumentale et technocratique est devenue envahissante, selon lui, et a eu pour résultat d'instrumentaliser de plus en plus l'éducation.

La conséquence logique de cette forme de rationalité aboutit à l'idée que le métier d'enseignant est une activité professionnelle technique spécialisée, bien que la dimension morale de l'éducation et la conception de l'enseignant comme "passeur intellectuel» soient, historiquement, au cœur de la tradition européenne de l'éducation. Sultana appelle les enseignants à se réapproprier cette vision plus large et à rejeter le rôle de «fournisseur, évaluateur et technicien» (p178). Perrenoud (1996) lance le même appel. Dans son esquisse du choix entre prolétarisation et professionnalisation auquel les enseignants sont confrontés dans de nombreuses sociétés développées, il affirme qu'ils peuvent choisir et même influencer le cours des choses mais qu'ils doivent, avant tout, définir clairement leur rôle pour pouvoir le négocier.

Les responsables politiques, en Angleterre comme dans d'autres pays, sont à l'évidence confrontés à un choix. L'enquête PACE, renforcée par d'autres enquêtes sur l'école primaire anglaise, laisse entendre que l'évolution du système éducatif anglais vers une technocratisation de l'enseignement à tous les niveaux (classe, établissement, contexte scolaire dans son ensemble) entrave le développement d'une collégialité réelle (Webb and Vulliamy, 1996; Hargreaves, 1991). Comme je l'ai signalé au début de l'article, cela confirme que la satisfaction professionnelle de l'enseignant et son implication, ou son engagement, se nourrissent de la diversité et du sentiment d'autonomie dans son travail (Rowan, 1994). Cela pose des questions essentielles sur la nature de l'enseignement et de l'apprentissage. Comme nous l'avons montré, les enseignants du primaire en Angleterre ont été confrontés au cours des dernières années à la question suivante: «est-ce cela l'enseignant que je veux être?» Nous devons à présent nous demander: "quelle sorte d'enseignant voulons-nous pour former les futurs citoyens de notre société?»

\section{BibLIOGRAPHIE}

ACKER S. (1999): The Realities of Teachers' Work: never a dull moment. London: Cassell.

ALEXANDER R. (1997): Policy and Practice in Primary Education. London: Routledge.

BALL S.J. (1994): Education Reform: a critical and post-structural approach. Buckingham: Open University Press. 
BALL S.J. (1999): Educational Reform and the Struggle for the Soul of the Teacher! Hong Kong, The Chinese University of Hong Kong.

BERNSTEIN B. (1996): Pedagogy, Symbolic Control and Identity. London: Taylor and Francis.

CROLl P., ABBOTT D., BROADFOOT P., OSBORN M. and POLLARD A. (1994): Teachers and Educational Policy: roles and models. British Journal of Educational Studies, 42 (2), 333-347.

CROLL P., ABBOTT D., BROADFOOT P., OSBORN M. and POLLARD A. (1994): Primary Teachers and Educational Policy. PACE Working Paper No. 21, presented at BERA Conference, Oxford. September.

CROLL P., ABBOTT D., POLLARD A., BROADFOOT P. and OSBORN M. (1994): Coercion or Compromise: how schools react to imposed change. PACE Working Paper No. 15, presented at AERA Conference, New Orleans. April.

GALTON M., HARGREAVES L., COMBER C., WALL D. and PELL A. (1999): Inside the Primary Classroom: 20 years on. London: Routledge.

GIPPS C.V., BROWN M., MCCALLUM B. and MCMANUS S. (1995): Intuition or Evidence? Teachers and National Assessment of Seven-Year-Olds. Buckingham: Open University Press.

HARGREAVES A. (1991): Contrived Collegiality: the micro-politics of teacher collaboration. Managing Change in Education. Bennett et al. London, Chapman/Open University.

HARGREAVES A. (1994): Changing Teachers, Changing Times: teachers' work and culture in the post-modern age. London: Cassell.

HELSBY G. and MCCULLOCH G. (eds) (1997): Teachers and the National Curriculum. London: Cassell.

JEFFREY B. and WOODS P. (1996): Feeling Deprofessionalised: the social construction of emotions during an OFSTED inspection. Cambridge Journal of Education, 26 (3), 325-343.

MENTER I., MUSCHAMP Y., NICHOLLS P., OZGA J. and POLLARD A. (1997): Work and Identity in the Primary School: a post-Fordist analysis. Buckingham: Open University Press.

NIAS J. (1989) : Primary Teachers Talking. London: Routledge.

NIAS J., SOUTHWORTH G. and YEOMANS R. (1989): Staff Relationships in the Primary School: a study of organisational cultures. London: Cassell.

NIXON J., MARTIN J., MCKEOWN P. and RANSON S. (1997): Towards a Learning Profession: changing codes of occupational practice within the new management of education. British Journal of Sociology of Education, 18 (1), $5-28$. 
OSBORN M., MCNESS E., BROADFOOT P., POLLARD A. and TRIGGS P. (2000) : What Teachers Do: Changing Policy and Practice in English primary schools. London: Cassell.

PERRENOUD P. (1996): The Teaching Profession Between Proletarianisation and Professionalisation: two models of change. Prospects, 26 (3), 509-529.

POLLARD A., TRIGGS P., BROADFOOT P., MCNESS E. and OSBORN M. (2000) : What Pupils Say: Changing Policy and Practice in English Primary Education. London: Cassell.

RICHARDS C. (1998): Curriculum and Pedagogy in Key Stage 2: a survey of policy and practice in small rural primary schools. The Curriculum Journal, 9 (3), 319-332.

ROWAN B. (1994): Comparing Teachers' Work With Work in Other Occupations: notes on the professional status of teaching. Educational Researcher. Aug-Sept.

SULTANA R.G. (1994): Conceptualising Teachers' Work in a Uniting Europe. Compare, 24 (2), 171-182.

TORRANCE H. and PRYOR J. (1998) Investigating Formative Assessment: teaching, learning and assessment in the classroom. Buckingham: Open University Press.

TROMAN G. (1996): The Rise of the New Professionals? The restructuring of primary teachers' work and professionalism. British Journal of Sociology of Education, 17 (4), 473-487.

WEBB R. and VUlliAMY G. (1996): A Deluge of Directives: conflict between collegiality and managerialism in the post-ERA primary school. British Educational Research Journal, 22 (4), 441-445.

WEBB R. and VULLIAMY G. (1996) Roles and Responsibilities in the Primary School: changing demands, changing practices. Buckingham: Open University Press.

WOODS P. (1995): Creative Teachers in Primary Schools. Buckingham: Open University Press.

WOODS P. and JEFFREY B. (1996) : Teachable Moments. Buckingham: Open University Press.

WOODS P. and JEFFREY B. (1997): Creative Teaching in the Primary National Curriculum. In HELSBY G. and McCULLOCH G. (eds), Teachers and the National Curriculum. London: Cassell.

WOODS P., JEFFREY B., TROMAN G. and BOYLE M. (1997) : Restructuring Schools, Reconstructing Teachers: responding to change in the primary school. Buckingham: Open University Press. 